\title{
Emotional pictures and sounds: a review of multimodal interactions of emotion cues in multiple domains
}

\author{
Antje B. M. Gerdes ${ }^{1}{ }^{*}$, Matthias J. Wieser ${ }^{2+}$ and Georg W. Alpers ${ }^{1,3}$ \\ ${ }^{1}$ Clinical and Biological Psychology, Department of Psychology, School of Social Sciences, University of Mannheim, Mannheim, Germany \\ 2 Department of Psychology, University of Würzburg, Würzburg, Germany \\ ${ }^{3}$ Otto-Selz Institute, University of Mannheim, Mannheim, Germany
}

\section{Edited by:}

Luiz Pessoa, University of Maryland, USA

\section{Reviewed by:}

Sarah Jessen, Max Planck Institute for Human Cognitive and Brain Sciences, Germany

Mathias Weymar, University of Greifswald, Germany

\section{${ }^{*}$ Correspondence:}

Antje B. M. Gerdes, Clinical and Biological Psychology, Department of Psychology, School of Social

Sciences, University of Mannheim, L13,17 D-68131 Mannheim, Germany e-mail:gerdes@uni-mannheim.de

${ }^{\dagger}$ Antje B. M. Gerdes and Matthias J. Wieser have contributed equally to this work.
In everyday life, multiple sensory channels jointly trigger emotional experiences and one channel may alter processing in another channel. For example, seeing an emotional facial expression and hearing the voice's emotional tone will jointly create the emotional experience. This example, where auditory and visual input is related to social communication, has gained considerable attention by researchers. However, interactions of visual and auditory emotional information are not limited to social communication but can extend to much broader contexts including human, animal, and environmental cues. In this article, we review current research on audiovisual emotion processing beyond face-voice stimuli to develop a broader perspective on multimodal interactions in emotion processing. We argue that current concepts of multimodality should be extended in considering an ecologically valid variety of stimuli in audiovisual emotion processing. Therefore, we provide an overview of studies in which emotional sounds and interactions with complex pictures of scenes were investigated. In addition to behavioral studies, we focus on neuroimaging, electroand peripher-physiological findings. Furthermore, we integrate these findings and identify similarities or differences. We conclude with suggestions for future research.

Keywords: multimodal emotion processing, emotional pictures, emotional sounds, audiovisual interactions, emotional scene stimuli, auditory stimuli

\section{INTRODUCTION}

In daily life, a wide variety of emotional cues from the environment reaches our senses. Typically, multiple sensory channels, for example vision and audition are integrated to provide a complete assessment of the emotional qualities of a situation or an object. For example, when someone is confronted with a dog, the evaluation of its potential dangerousness or friendliness will be more effective if visual (e.g., big vs. small dog; tail wagging or not) and auditory information (growling vs. friendly barking) can be integrated. While some of the information carried in either one of the channels may be redundant, the channels may also interact; i.e., a fierce bark may boost visual attention to the dog's bared teeth.

Despite the obvious relevance of multimodal perception in everyday life, emotion research has typically only investigated unimodal cues - with an apparent emphasis on visual stimuli. To cope with (a) limited processing capacities within a sensory modality and (b) the need to detect information which is relevant for survival, emotionally relevant cues have been suggested to modulate attention and selectively enhance perception (Vuilleumier, 2005; Pourtois et al., 2012).

Indeed, for the visual domain it has been shown that emotional cues - especially with threatening, but also with appetitive content - are preferentially processed in very early sensory areas (Schupp etal., 2003b; Pourtois et al., 2005; Gerdes et al., 2010). Emotional pictures influence perceptual processing and attract enhanced attention (Öhman and Wiens, 2003; Alpers and Pauli, 2006; Alpers and Gerdes, 2007; Gerdes et al., 2008, 2009; Stienen etal., 2011; Pourtois etal., 2012; Gerdes and Alpers, 2014).
Furthermore, distinct and intensive behavioral responses, physiological reactions, and brain activations are robustly evoked by emotional pictures (e.g., Lang et al., 1998; Neumann et al., 2005; Alpers etal., 2011; Eisenbarth etal., 2011; Plichta et al., 2012). According to Lang (1995) and Lang et al. (1998), the emotional response system is founded on an appetitive and defensive motivational system. Emotional states reflect these basic motivational systems and can be described in terms of affective valence and arousal. For example, a number of physiological measures are shown to covary with the valence or arousal of emotional cues: electromyographic (EMG) activity, heart rate responses and the startle reflex were shown to be sensitive to valence, whereas skin conductance and slow cortical responses are more sensitive to arousal (for more elaborative reviews on the processing of emotional pictures see, e.g., Bradley and Lang, 2000b; Brosch et al., 2010; Sabatinelli etal., 2011). Generally, enhanced processing gains of emotional cues may help individuals to quickly initiate adequate approach or avoidance behavior and therefore increases the chance of survival or well-being (Lang et al., 1997).

On the neural level the amygdala has long been identified as a key structure of emotional detection both in humans and animals (for reviews and meta-analysis see LeDoux, 2000; Phan et al., 2002; Costafreda et al., 2008; Armony, 2013). Relevant for the present context, via the thalamus the amygdala receives input not only from the visual modality but from all senses (Nishijo et al., 1988; Amaral et al., 1992; Amaral, 2003). The amygdala is instrumental in the relevance detection for biologically relevant 
cues and has been documented to operate independently from the sensory modality which conveyed the information (Armony and LeDoux, 2000; Sander et al., 2003; Zald, 2003; Öhman, 2005; Stekelenburg and Vroomen, 2007; Scharpf et al., 2010; Armony, 2013). There is empirical evidence that the amygdala processes, e.g., emotional visual (Royet et al., 2000; Phan et al., 2002) and auditory cues (Fecteau et al., 2007; Klinge et al., 2010), as well as olfactory (Gottfried et al., 2002), and gustatory cues (O'Doherty et al., 2001).

Despite the evidence that visual and auditory emotion processing recruits similar brain structures, research on emotional auditory information and on multimodal cues is relatively scant. Until recently, this research field has mainly examined multimodal integration in social communication, i.e., face-voice stimuli (for a recent review see Klasen et al., 2012).

\section{FACE-VOICE INTERACTION}

From studies using combined face-voice stimuli, we know that audiovisual integration can facilitate and improve perception, even beyond the emotion effects within each separate channel. Emotion recognition is improved in response to multimodal compared to unimodal face-voice stimuli (Vroomen et al., 2001; Kreifelts et al., 2007; Paulmann and Pell, 2011). Furthermore, the identification of an emotional facial expression is facilitated when the face is accompanied by an emotional congruent voice and the evaluation of emotional faces is biased toward the valence of a simultaneously presented voice (de Gelder and Vroomen, 2000; de Gelder and Bertelson, 2003; Focker et al., 2011; Rigoulot and Pell, 2012). Such interactions appear to be independent of attentional allocation, i.e., even when participants are instructed to pay attention to only one sensory modality, emotional information of a concurrent but non-attended sensory channel influences the processing of the attended modality (Collignon et al., 2008). Likewise, if emotional faces and voices tap the same emotional valence (emotional congruency), they were processed faster than emotionally incongruent stimulus pairs or unimodal stimuli even when the attentional focus was explicitly directed to the faces or to the voices (Focker et al., 2011). Furthermore, this cross-modal influence was independent of a demanding additional task which had to be performed in parallel (Vroomen et al., 2001).

On the neuronal level, face-voice integration can occur at early perceptual stages of stimulus processing (for more specific information see the review of Klasen et al., 2012). Furthermore, specific brain areas such as superior and middle temporal structures and the fusiform gyrus, as well as parts of the emotion processing network including the thalamus, amygdala, and insula are consistently involved in emotional face-voice integration (see also Klasen et al., 2012). Taken together, the integration of emotional faces and voices is an important part of social interaction and the prioritized processing and early integration of emotional face-voice pairs is an essential feature of social cognition (de Gelder and Vroomen, 2000).

\section{BEYOND EMOTIONAL "FACE-VOICE" PROCESSING}

In this review, we argue that a broader variety of stimuli should be considered in audiovisual emotion processing. While stimuli which are directly linked to human communication may represent an important subset of cues, a meaningful extension to existing concepts of multimodality should be carried forward by considering other domains as well. In this review we focus on visual and auditory cues across a wide range of semantic categories.

We start out with a short overview of studies mainly from the visual domain which focus on differences between emotional human communication and scene processing. This section demonstrates that the processing of communication vs. scene stimuli recruits different brain structures and elicit distinct electroand peripher-physiological responses. Thus, we argue that a distinction between those kinds of stimuli in multimodal emotion research is important and useful.

Because research on emotional sounds is much less frequent than research on emotional pictures we give a short overview of how emotional complex sounds can affect self-report ratings, physiological responses, and brain processes, and then summarize similarities and differences between emotional sound and picture processing.

Furthermore, we review studies which investigate how emotional information in one sensory modality can influence information processing of neutral cues in another sensory modality. Finally, we will summarize the existing studies that focus on interactions of the concurrent processing emotional visual and emotional auditory cues beyond faces and voices. We will conclude with a short summary and an outlook on research questions where the application of multimodal stimuli is particular interesting.

\section{HUMAN COMMUNICATION VS. EMOTIONAL SCENE STIMULI}

Beyond face-voice integration, there are only few studies which focus on audiovisual interactions in emotion research. On the one hand, quite similar to face-voice interactions, some studies investigated multimodal integration in human communication with regard to bodily gestures and vocal expressions (Stekelenburg and Vroomen, 2007; Jessen and Kotz, 2011; Stienen et al., 2011; Jessen et al., 2012). On the other hand, there are several studies which examine influences of music on the processing of visual stimuli (e.g., Baumgartner et al., 2006; Logeswaran and Bhattacharya, 2009; Marin et al., 2012; Hanser and Mark, 2013; Arriaga et al., 2014). Because music is man-made, many theorists claim, that it is in essence another form of human communication. Therefore, music may be more similar to the communicative channels described above than to other naturally occurring sounds. In a similar vein, it has been argued that music has no obvious survival value (Juslin and Laukka, 2003).

Generally, it has been demonstrated that a fast and effective integration of stimuli across different modalities is necessary in several (survival-relevant) contexts. These contexts are certainly not limited to social situations. Examples for non-social situations of high biological significance may be a growling bear, swirling wasps, or an approaching thunderstorm. In these examples congruent visual and auditory information is transmitted (to the individual), whose prioritized processing may help the organism to survive. The first obvious advantage of multimodal information is that uncertainties in one sensory channel can be easily compensated and complemented by the other channel. In addition, even if the (emotional) information conveyed by ear and eye is obviously redundant, multisensory integration effects 
can be clearly distinguished from redundancy effects within one modality. Again, the only empirical evidence supporting this claim stems from research on face-voice pairings. Redundant emotional information within the same modality leads to (post-) perceptual interferences shown by a lesser accuracy and longer response latencies within an emotional expression discrimination task. In contrast, congruent information of faces and voices was integrated early and pre-attentive with a clear perceptual benefit (Pourtois and Dhar, 2013). Thus, both senses supplement each other to create a distinct multisensory emotional percept (for a detailed discussion on stimulus redundancy; Pourtois and Dhar, 2013). Compared to information from face and voice, in many natural situations, not only concordant but also unrelated information during the same event can be conveyed by the different sensory channels (e.g., the sound of an emergency siren while watching children playing on a playground).

Empirical evidence for multifaceted differences between human communication and scene stimuli comes from the visual domain. On the peripher-physiological level, Alpers et al. (2011) showed that emotional scenes and faces were rated similarly, but the pattern of physiological responses measured by startle reflex, heart rate, and skin conductance was different. Startle responses to emotional scenes were modulated by valence with lowest amplitudes for positive, intermediate for neutral, and highest amplitudes for negative scenes, whereas the startle response was similarly enhanced in response to negative and positive faces. Furthermore, negative scene picture show a greater heart rate deceleration compared to neutral and positive scene; whereas negative and positive faces were followed by heart rate deceleration. These results indicate that scenes result in a valence based modulation, faces an arousal based modulation. In contrast, the skin conductance was arousal-modulated for the scene pictures with higher responses to the negative pictures and a valence-specific modulation for the faces with highest responses to positive faces. The facial EMG showed similar responses to both contents, but responses were slightly greater in response to scenes. Likewise comparing emotional faces and scenes, a recent study (Wangelin et al., 2012) showed that emotional scenes evoked stronger reactions in autonomic, central, and reflex measures in comparison to faces. In a meta-analysis (Sabatinelli et al., 2011), it was shown that emotional scenes elicited activation in occipital regions, the pulvinar, and the medial dorsal nucleus of the thalamus whereas the fusiform gyrus and the temporal gyrus were specifically activated in response to faces. Thus, measured emotion effects can strongly depend on the presented class of stimuli. Particularly, in emotion research one may argue that while face stimuli certainly convey emotional information, they do not necessarily elicit emotions in the observer (Ruys and Stape, 2008). Taken together, evidence from the visual domain clearly highlights the importance of a separate consideration of face and scene stimuli in emotion research. Analogous to that, for the auditory domain there is empirical evidence that human vocal and non-vocal sounds generally have different electrophysiological correlates and can elicit distinct responses in auditory regions (Meyer et al., 2005; Bruneau et al., 2013).

Thus, investigations with affective scene cues which have been consistently demonstrated to elicit emotional responses on behavioral and physiological levels (for emotional sounds see below) are needed to answer research questions about multi-modal emotion processing beyond face-voice stimuli.

\section{EMOTIONAL SOUND PROCESSING}

Compared to visual cues, sounds are still investigated only rarely. It is likely that this is due to the development of research traditions according to practical considerations rather than a reflection of the relative importance of auditory cues. Compared to pictures, sounds may be somewhat less amenable to experimental designs in the laboratory. However, sounds can clearly prompt strong emotional responses as has been shown in a large internetbased survey (Cox, 2008b). The development of the International Affective Picture System (IAPS; Lang et al., 2008) has been followed by a similar collection of sounds, the International Affective Digitized Sounds (IADS; Bradley and Lang, 2007) - a series of naturally occurring human, non-human, animal, and environmental sounds (e.g., bees buzzing; applause, explosions). Existing research on emotional sound processing (beyond voices) has been almost exclusively used this series as stimulus material (see below). In two experiments by Bradley and Lang (2000a), it was shown that valence and arousal ratings of these sounds were comparable to affective pictures from the IAPS. Furthermore, emotionally arousing sounds were also remembered better than neutral sounds in a free recall task. On a physiological level, emotionally arousing sounds elicit larger electrodermal activity which is generally known to be sensitive to the arousal of emotional stimuli (Bradley and Lang, 2000a). In comparison to pleasant sounds, the startle response to unpleasant sounds is enhanced and unpleasant sounds were accompanied by stronger corrugator activity and larger heart rate deceleration. This suggests that unpleasant sounds reliably activate the defensive motivational system (Bradley and Lang, 2000a). Another study showed that emotional sounds were accompanied by larger pupil dilatation which is an index of higher autonomic activity elicited by emotion (Partalaa and Surakka, 2003). Electrophysiological results suggest that aversive auditory cues (as, e.g., squeaking polystyrene) compared to neutral sounds were accompanied by a more pronounced early negativity and later positivity of event-related brain potentials as a measure of enhanced allocation of attention (Czigler et al., 2007) similar to what has been observed in emotional pictures (Schupp et al., 2003a). In contrast, unpleasant environmental sounds capture enhanced attention (shown by increased P3a amplitudes) but do not influence earlier components of perceptual processing (Thierry and Roberts, 2007). Similarly, two fMRI studies (Scharpf et al., 2010; Viinikainen et al., 2012) measured brain activation in response to emotional sounds from the IADS. Both studies showed that emotional sounds elicited strong activation in the amygdala compared to neutral sounds. Specifically, Viinikainen et al. (2012) showed that there was a quadratic U-shaped relationship between the sound valence and brain activation in the medial prefrontal cortex, auditory cortex, and amygdala with the weakest activation for neutral and increased activation for unpleasant and pleasant sounds.

Importantly, in an fMRI-study (Kumar et al., 2012) there was evidence that the amygdala encodes both the acoustic features of an auditory stimulus and the perceived unpleasantness. Specifically, 
acoustic features modulate effective connectivity from auditory cortex to the amygdala whereas valence modulates the effective connectivity from amygdala to the auditory cortex. Thus, control of acoustic features is of specific importance in research on emotional sounds.

A recent study from our research group investigated the processing of emotional sounds from the IADS within the auditory cortex (Plichta et al., 2011). Because fMRI scanner noise can interfere with auditory processing we used near-infrared spectroscopy (NIRS) which is a silent imaging method. In addition, the sound material was carefully controlled for several physical parameters such as loudness and spectral frequency. Unpleasant and pleasant sounds enhanced auditory cortex activation as compared to neutral sounds suggesting that the enhanced activation of sensory areas in response to complex emotional stimuli is apparently not restricted to the visual domain.

Further support for this observation comes from an MEGStudy investigating the influence of emotional content of complex sounds on auditory-cortex activity, both during anticipation and hearing of emotional and neutral sounds (Yokosawa et al., 2013). Indeed, during the hearing as well as during the anticipation period, unpleasant and pleasant sounds evoked stronger responses within the auditory cortex than neutral sounds.

In sum, there is now considerable evidence that complex highly arousing pleasant and unpleasant sounds are processed more intensively on a peripheral as well as on early sensory processing levels. Thus, using standardized emotional sounds (e.g., the IADS) can serve as a useful research tool to elicit emotions and investigate emotion processing.

\section{EMOTIONAL SOUNDS AND PICTURE PROCESSING: SIMILARITIES AND DIFFERENCES}

Generally, emotional sound and picture processing is very comparable. The pattern of behavioral and physiological and electrophysiological reactions elicited by emotional sounds is comparable to emotional pictures (Bradley and Lang, 2000a; Schupp et al., 2003a; Czigler et al., 2007). However, there is some evidence that reactions to emotional sounds are weaker (Bradley and Lang, 2000a) and occur later (Thierry and Roberts, 2007). On the neuronal level, both emotional sounds and pictures gain privileged access to processing resources in the brain. Brain responses to visual, auditory, and olfactory stimuli were measured with PET showing for all three modalities, that all emotional stimuli activated the orbitofrontal cortex, the temporal pole, and the superior frontal gyrus (Royet et al., 2000). In addition, Scharpf et al. (2010) compared brain activation to sounds with responses to IAPS pictures. Independent of the sensory modality, the amygdala, the anterior insula, the STS, and the OFC showed increased activation during the processing of emotional as well as social stimuli. Also comparing brain activation to emotional pictures from the IAPS and to emotional sounds from the IADS, increased amygdala activity in response to both, emotional pictures and sounds were reported (Anders et al., 2008). Differentially, the left amygdala was sensitive to the valence of pictures and negative sounds whereas the right amygdala responded to the valence of positive pictures. A recent study directly aimed at investigating whether affective representations differ with sensory modality (Shinkareva et al., 2014).
Therefore, emotional picture and sound stimuli were presented in an event-related fMRI experiment. The results mainly provide evidence for a modality specific instead of a modality-general valence processing effect. Specifically, voxels were identified that were sensitive to the valence of pictures within the visual modality, as well as voxels that were sensitive to the valence of sounds within the auditory modality, but no voxels that were sensitive to valence across the two modalities.

To sum up, emotional pictures and sounds mainly elicit similar reactions on the level of self-report, behavioral, physiological, and neuronal - both types of stimuli strongly activate appetitive and defensive motivational circuits (Bradley and Lang, 2000a; Lang and Bradley, 2010). The reported processing differences (e.g., intensity of reaction, laterality effects, and timing) might be - at least partly - the result of methodological differences and different stimulus characteristics which are obvious between sounds and pictures (e.g., the dynamic nature of sounds). Thus, to account for such differences and to interpret potential processing differences, systematic and direct comparisons between emotional picture and sound processing with well controlled and (physically) comparable stimuli (e.g., conditioned stimuli) are urgently needed.

\section{AUDIO-VISUAL INTERACTIONS INTERACTION OF VISUAL AND AUDITORY PROCESSING}

Generally and beyond the emotional domain, it is well established that visual information can foster early stages of auditory processing and vice versa. For example, auditory speech perception can be strongly influenced by the viewing of visual speech stimuli on the perceptual (McGurk and MacDonald, 1976) as well as on the neuronal level (see, e.g., Kislyuk et al., 2008). Likewise, visual processing can be strongly altered by concurrent sounds even at the earliest stage of cortical processing (Bulkin and Groh, 2006; Shams and Kim, 2010). Based on these findings it seems plausible that such interaction may also occur when emotional information is conveyed by (at least) one of the sensory channels. Indeed, a small but growing number of studies suggest that (emotion) processing in the auditory system can be influenced by (non-related) emotional information coming from the visual modality and vice versa (see below).

\section{INTERACTION OF EMOTIONAL VISUAL AND NON-EMOTIONAL AUDITORY PROCESSING}

On a behavioral level, a recent study investigated the influence of emotional IAPS pictures on the classification of high and low pitch tones but did not find an effect of picture valence on the auditory classification (Ferrari et al., 2013).

On the physiological level, it is well-known that emotional visual stimuli can modulate the acoustic startle reflex elicited by loud, abrupt, and unexpected sounds: negative pictures enhance, positive pictures dampen the blink magnitude in response to the unexpected sound (e.g., Lang et al., 1990). Moreover, the electrocortical response to the acoustic startle probe (P3 component) was also found to be modulated by the arousal of the emotional pictures in the foreground with smaller amplitudes for high arousing pictures (Keil et al., 2007).

Regarding electrophysiological responses, the presentation of unpleasant pictures has a significant impact on event-related 
potentials of the EEG to strongly deviant tones. During the presentation of unpleasant pictures, high deviant tones elicited larger $\mathrm{N} 1$ and $\mathrm{P} 2$ responses than during the presentation of pleasant pictures which was interpreted as a sensitization to potentially significant deviant events (N1) and enhanced attention (P2) to regular external events (Sugimoto et al., 2007). Similarly, auditory novelty processing was enhanced by negative IAPS pictures. Participants had to judge (emotional) picture pairs as equal or different while ignoring task irrelevant sounds. During the presentation of negative IAPS-pictures, novel sounds compared to the standard tone provoked enhanced distraction effects shown on the behavioral level as well by the modulation of event-related potentials (enhanced early and late novelty P3; Dominguez-Borras et al., 2008a,b). In a similar vein, pleasant pictures were shown to modulate auditory information processing such that they significantly attenuated the Mis-Match-Negativity (MMN) in response to a change within an auditory stimulus stream. Thus, pleasant pictures can be seen as a kind of safety signals and probably reduce the need for auditory change detection (Surakka et al., 1998).

Further support for crossmodal influences of emotion is provided by a MEG study showing that unpleasant pictures diminish auditory sensory gating in response to repeated neutral tones as an index of neuronal habituation (Yamashita et al., 2005). Presenting neutral tones subsequent to emotional pictures, another study showed that neutral tones prompted larger ERP amplitudes (N1 and N2) when emotional relative to neutral pictures were presented before, indicating enhanced enhance attention and orienting toward neutral tones encoded in the context of emotional scenes (Tartar et al., 2012). All studies reported above indicate that emotional visual information can enhance auditory processing. However, the question arises whether increasing demands of an auditory task might interfere with the processing of emotion in the visual domain. This may be due to competition for limited processing resources, a process which has been documented for competing emotional pictures within the same modality (Schupp et al., 2007). However, the processing of emotional IAPS pictures was not modulated by an additional auditory detecting task with increasing complexity (Schupp et al., 2008). Thus, emotion processing in the visual domain was not affected by task demands in the auditory modality. This finding is in line with the multiple resource theory which assumes that each sensory modality has separate pools of (attentional) resources (Wickens, 2002).

Taken together, emotional cues in the visual domain are able to enhance concurrent as well as subsequent auditory processing even at very early processing stages with no or low costs for emotion processing.

\section{INTERACTION OF EMOTIONAL AUDITORY AND NON-EMOTIONAL VISUAL PROCESSING}

To the best of our knowledge, evidence that emotion cues from the auditory modality can also influence non-emotional (basic) visual information processing is nearly missing. One study investigated the influence of emotional sounds on visual attention in a spatial cueing paradigm (Harrison and Davies, 2013). Here, non-speech environmental sounds from the IADS were presented spatially matched to the locations of subsequent visual targets. Indeed, results show for right-sided targets that neutral and positive sounds elicited faster responses to valid trials (where the sound and the visual target were presented on the same side) compared to invalid trials. In contrast, after negative sounds, the reaction time to valid trials was slower suggesting faster attentional disengagement from negative sounds.

Another study used spoken emotional and neutral words that were followed by a visually presented neutral target word (Zeelenberg and Bocanegra, 2010). It was found that identification of a masked visual word was improved by preceding spoken emotional words as compared to neutral ones. These findings can be interpreted as first evidence that affective sounds may influence at least subsequent visual (word) processing. However, much more studies are needed in which the effects of emotional sounds on concurrent and subsequent visual processing are investigated.

\section{INTERACTION OF EMOTIONAL AUDITORY AND EMOTIONAL VISUAL PROCESSING}

We assume that audio-visual interactions are possible in both directions. As reviewed above, emotional visual as well as auditory information are preferentially and intensively processed. Thus, one can expect that audio-visual interactions of emotional stimuli occur even stronger if emotional information is conveyed by both modalities. Regarding that question, self-report data of an internet-based survey suggest that sound stimuli were significantly perceived as more horrible when they were accompanied by pictures that show associated information (e.g., the sound of a crying baby combined with a picture of a crying baby) compared to pictures with unassociated pictures (Cox, 2008a). Using affective IADS sounds; Scherer and Larsen (2011) found significant cross-modal priming effects for negative sound primes on emotional visual word targets. Experimentally, self-report (valence and arousal) and physiological variables were measured in response to unimodal and bimodal presented emotional sounds and pictures in a within subjects design. Unpleasant and pleasant stimuli had similar effects on self-report, heart rate, heart rate variability, and skin conductance with no effect of stimulus modality. Contrary to expectations, bimodal presentation with congruent visual and auditory stimuli did not enhance the effects (Brouwer et al., 2013).

In a similar vein, we recently conducted an EEG-study in which unpleasant, pleasant, and neutral IAPS pictures were preceded by unpleasant, pleasant, and neutral IADS sounds. Ratings and electrophysiological data suggest that (emotional) sounds clearly influence emotional picture processing (Gerdes et al., 2013). We could demonstrate that audiovisual pairs with pleasant sounds and pictures were rated as more pleasant than pleasant pictures only. In addition, valence congruent audiovisual combinations were rated as more emotionally as other incongruent combinations. Electrophysiological measures showed that ERP amplitudes ( $\mathrm{P}$ and $\mathrm{P} 2$ ) were enhanced in response to all pictures which were accompanied by emotional sounds compared to pictures with neutral sounds. These findings can be interpreted as evidence that emotional sounds may unspecifically increase sensory sensitivity or selective attention (P1, P2) to all incoming visual stimuli. Most importantly, unpleasant pictures with pleasant sounds prompted larger ERP amplitudes (P1 and P2) compared to unpleasant pictures with unpleasant sounds. The reduced amplitudes in response to congruent sound-picture pairs suggest that the processing of 
unpleasant pictures is facilitated (i.e., less processing resources are needed) when they were preceded by congruent unpleasant sounds.

Taken together, the above mentioned studies strongly suggest that emotion processing in one sensory modality can strongly affect emotion processing of another modality during very early stages of neuronal processing, as well as on the self-report level.

For a short overview of the here reviewed studies see Table 1.

\section{CONCLUSION AND FUTURE DIRECTIONS}

As the review summarized at the beginning, the majority of research on unimodal auditory emotion processing provides clear evidence that complex emotional auditory stimuli (mainly investigated with IADS) elicit similarly intensive emotional reactions on behavioral, physiological, and on neuronal levels as traditionally used complex visual emotional scenes. Furthermore, emotional cues from both modalities guide selective attention and receive enhanced processing. This preferential processing can even alter (emotional) information processing in other sensory channels. Specifically, there is evidence that the processing of complex emotional information in one sensory modality can strongly affect (emotion) processing of another modality during very early stages of neural processing, as well as self-reported emotions, and that these effects are bidirectional. As hitherto existing research mainly focused on stimuli of human communication such as faces and voices, the here reviewed work expands the concept of multimodality to a broader variety of human, animal, and environmental cues. However, as this review also indicates research in this field is in its infancy. Consequently, to carve out similarities and differences between the different classes of stimuli, disentangling emotional from social relevance (see, e.g., Bublatzky et al., 2014b), and the impact on audiovisual combinations are promising areas of future research.

From a methodological viewpoint, complex scene stimuli additionally offer the opportunity to separate effects of semantic (or contextual) and emotional (in)congruence in multimodality which is usually confounded in face-voice pairings. Similarly important is the dissociation of (task) difficulty from incongruence in multimodal emotion integration (Watson et al., 2013). Furthermore, effects of presentation order and timing of the stimuli should be investigated systematically, because such methodological differences seem partly responsible for inconsistent findings (see Jessen and Kotz, 2013). Also important, research on multimodality should strongly reveal effects simply caused by stimulus redundancy or by intensity amplification in contrast to inherent multimodality (Pourtois and Dhar, 2013). In order to investigate the (interactive) effects of sounds, more diverse stimulus sets would be highly welcome. Specifically, research on influences of emotional sounds on (subsequent) visual processing is pending. To account for physical differences of emotional picture and sounds, investigations with well controlled and (physically) comparable stimuli (e.g., with instructed fear or conditioning procedures) are urgently needed (see, e.g., Bröckelmann et al., 2011; Bublatzky et al., 2014a).

Regarding the impact of attention and automaticity, there has been a controversy for unimodal emotional cues whether they are processed outside of explicit attention or whether attentional disengagement can reduce neural responses and behavioral output (Pessoa, 2005). For example some studies claimed that visual threat cues activate the amygdala independently from attentional allocation (Straube et al., 2006) or that attentional distraction actually resulted in reduced activation (Pessoa et al., 2002; Alpers et al., 2009). We are not aware of systematic investigations of multimodal emotional stimulation and variations in attention to one or multiple channels. Interestingly, multimodal presentations may provide a particularly fruitful avenue for this debate because it is possible to attend to one channel and ignore the other.

With regards to other electrophysiological indices of preferential processing and attention, the $\mathrm{N} 2 \mathrm{Pc}$ or steady-state visual evoked potentials (ssVEPS) which are established in the visual domain (Wieser et al., 2012b; Weymar et al., 2013), would lend themselves for the examination of sound as well as for audio-visual cues and interactions. For example, it would be interesting to see how the N2pc as an index of visual-spatial attention to a salient stimulus in visual search paradigms is modulated by concurrent (emotional) sounds. Also, the influence of emotional sounds on sustained attentional processes (as measured by ssVEPS) would be an interesting research question. The use of different paradigms would help inform us about the different stages at which presumably emotional cues from different modalities interact. Recently, novel paradigms have been introduced to examine the behavioral output of preferentially processed emotional cues (see, e.g., Pittig et al., 2014). If integrated multimodal cues result in a more intensive emotional experience (and neural processing), this may also result in more pronounced behavioral consequences.

To make research more ecological valid and to evolve a broader and more complete concept of emotional multimodality, future research should not only concentrate on audiovisual emotion processing but should also incorporate cues from other sensory channels as, e.g., olfactory (Pause, 2012; Adolph et al., 2013), somatosensory (Francis et al., 1999; Gerdes et al., 2012; Wieser et al., 2012a, 2014), or gustatory signals (O'Doherty et al., 2001; Tonoike et al., 2013) which are also known to elicit emotional reactions and may interact with information processes of other modalities.

Another issue that is of great theoretical and practical importance is the consideration of different populations in the context of multimodal emotion processing. From a clinical perspective, the consideration of multimodal emotional processing is promising for the understanding of several mental disorders (e.g., anxiety disorders). Here, contextual (multimodal) information contributes to the acquisition and maintenance of the disorder (Craske et al., 2006). Accordingly, it has been argued elsewhere that for example in research on social anxiety disorder a crossmodal perspective may help to gain a more complete and ecological picture of cognitive biases and understand fundamental processes underlying biases in social anxiety (Peschard et al., 2014). Altogether, explicit knowledge on multimodal integration and interaction processes can improve the understanding of emotion processing (deficits) and consequently may help to optimize therapeutic approaches (see Taffou et al., 2012; Maurage and Campanella, 2013).

For the most part, the here reviewed interactions between emotional stimuli in the two senses can be explained on the background of the motivational priming theory (Lang, 1995). 

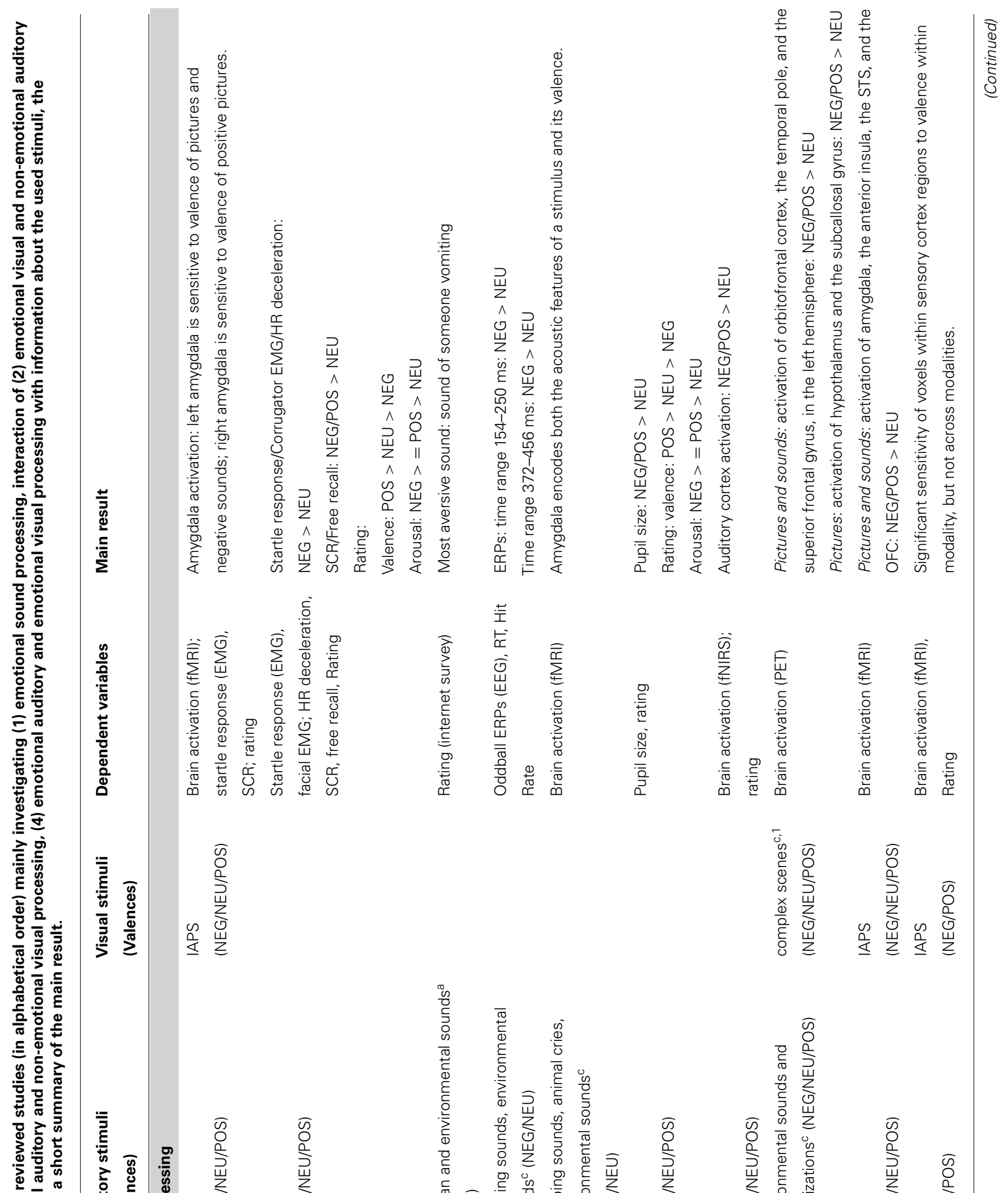

†
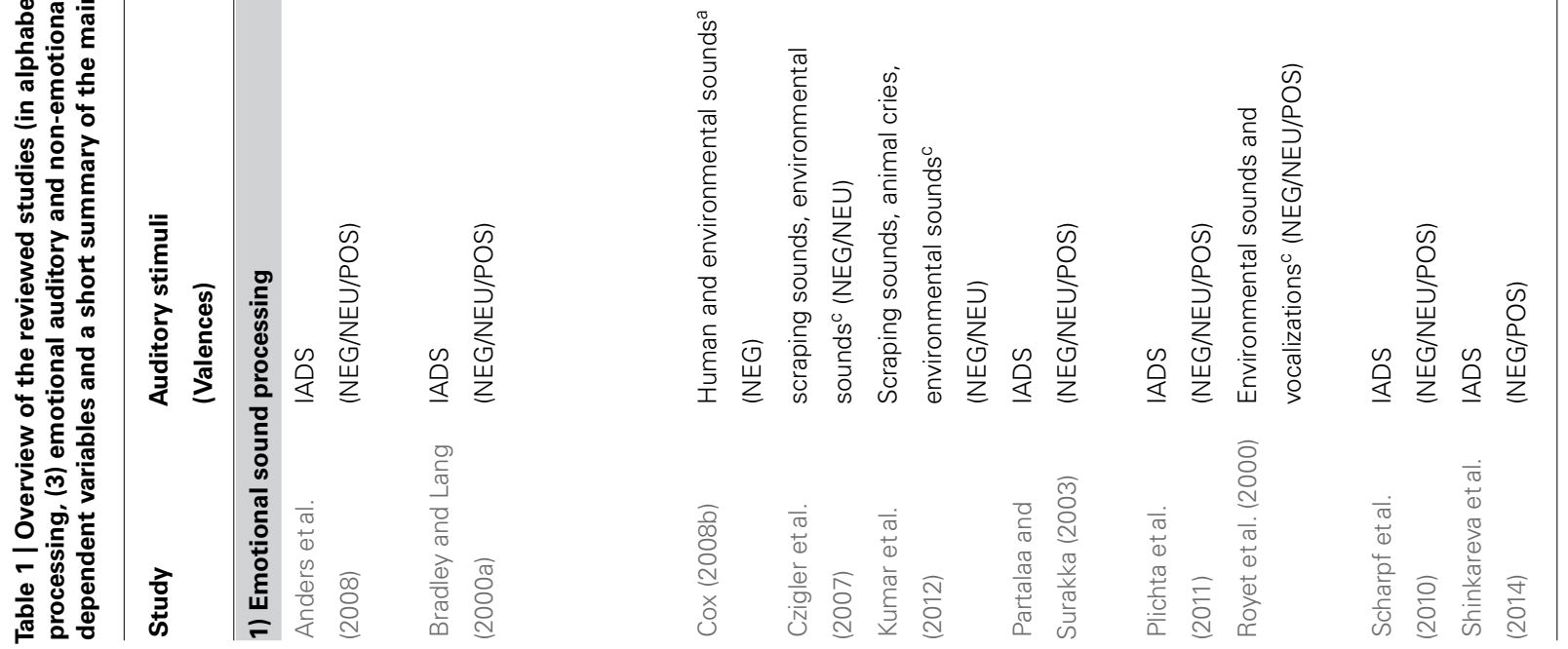


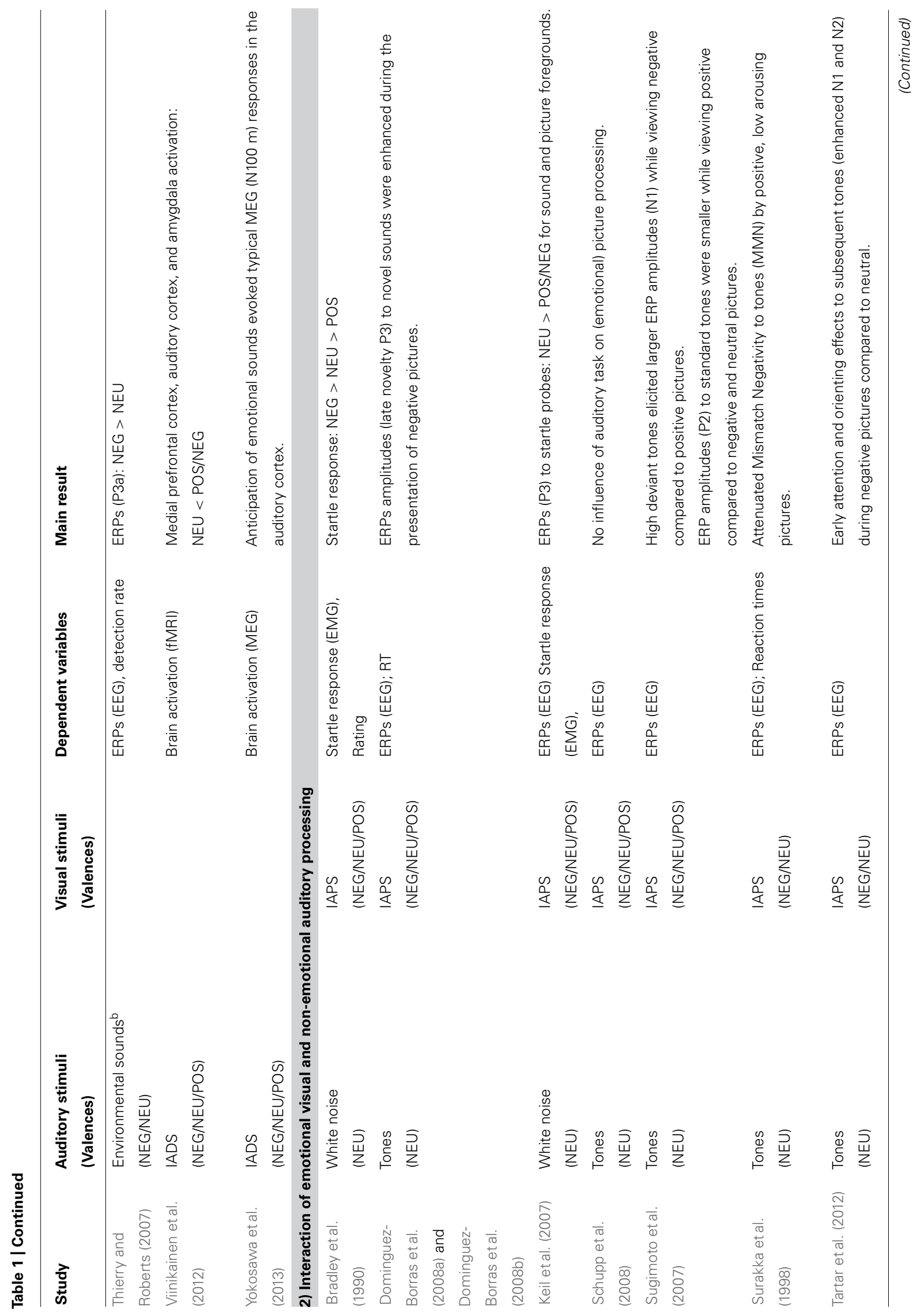




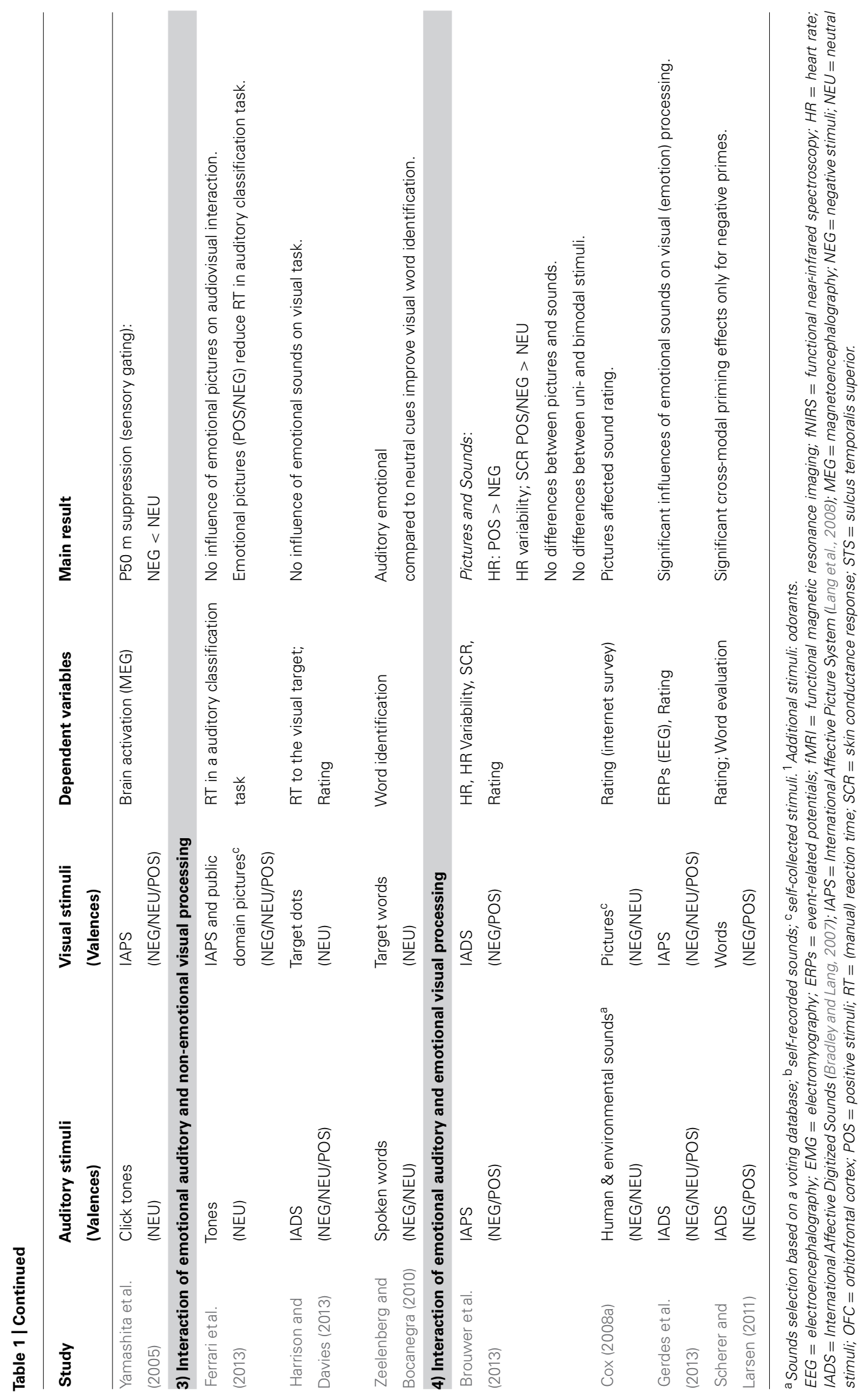


According to that theory, emotion is considered to be organized around two motivational systems, one appetitive, and one defensive. These systems have evolved to mediate behavior that either promote or threaten physical survival (Lang et al., 1997).

Independent of the sensory modality, emotional information is thought to activate the appetitive or defensive motivational system. Consequently, the engaged motivational system modulates other (brain) processing operations which means that (perceptual) processing of other emotional information can be facilitated or inhibited. These modulatory effects are shown crossmodally, thus, there also seem to be independent of the stimulus modality (see, e.g., Bradley et al., 1990; Lang et al., 1998).

Taken together, the motivational priming theory is able to explain audiovisual interactions of emotional information. However, the motivational priming theory does not make any assumptions of how multisensory emotional inputs are combined and integrated. Actually, no specific model exists which accounts for the integration of multisensory emotional information. Generally, one can assume that multisensory integration of emotional information follows similar principles as multisensory integration of other types of complex information (see, e.g., Stein and Meredith, 1993; de Gelder and Bertelson, 2003; Ernst and Bülthoff, 2004; Spence, 2007). Within the scope of the motivational priming theory, motivated attention might influence the efficiency of this integration processes. However, the development and the systematic testing of a specific theoretical framework for multimodal emotion processing is definitely one of the next important future challenges.

\section{ACKNOWLEDGMENTS}

This work was supported by the German Research Society (DFG; GE 1913/3-1-FOR 605). In addition, we thank Michael M. Plichta for his valuable feedback and discussions.

\section{REFERENCES}

Adolph, D., Meister, L., and Pause, B. M. (2013). Context counts! Social anxiety modulates the processing of fearful faces in the context of chemosensory anxiety signals. Front. Hum. Neurosci. 7:283. doi: 10.3389/fnhum.2013.00283

Alpers, G. W., Adolph, D., and Pauli, P. (2011). Emotional scenes and facial expressions elicit different psychophysiological responses. Int. J. Psychophysiol. 80, 173-181. doi: 10.1016/j.ijpsycho.2011.01.010

Alpers, G. W., and Gerdes, A. B. M. (2007). Here is looking at you: emotional faces predominate in binocular rivalry. Emotion 7, 495-506. doi: 10.1037/15283542.7.3.495

Alpers, G. W., Gerdes, A. B. M., Lagarie, B., Tabbert, K., Vaitl, D., and Stark, R. (2009). Attention and amygdala activity: an fMRI study with spider pictures in spider phobia. J. Neural Transm. 116, 747-757. doi: 10.1007/s00702-008-0106-8

Alpers, G. W., and Pauli, P. (2006). Emotional pictures predominate in binocular rivalry. Cogn. Emot. 20, 596-607. doi: 10.1080/02699930500282249

Amaral, D. G. (2003). The amygdala, social behavior, and danger detection. Ann. N. Y. Acad. Sci. 1000, 337-347. doi: 10.1196/annals.1280.015

Amaral, D. G., Price, J. L., Pitkanen, A., and Carmichael, S. (1992). "Anatomical organization of the primate amygdaloid complex," in The Amygdala, ed. J. P. Aggleton (New York: Wiley-Liss), 1-66.

Anders, S., Eippert, F., Weiskopf, N., and Veit, R. (2008). The human amygdala is sensitive to the valence of pictures and sounds irrespective of arousal: an fMRI study. Soc. Cogn. Affect. Neurosci. 3, 233-243. doi: 10.1093/scan/nsn017

Armony, J. L. (2013). Current emotion research in behavioral neuroscience: the role(s) of the amygdala. Emot. Rev. 5, 104-115. doi: 10.1177/1754073912457208

Armony, J. L., and LeDoux, J. (2000). "How danger is encoded: toward a systems, cellular, and computational understanding of cognitive-emotional interactions in fear," in The New Cognitive Neurosciences, 2nd edn, ed. M. S. Gazzaniga (Cambridge, MA: The MIT Press), 1067-1079.

Arriaga, P., Esteves, F., and Feddes, A. R. (2014). Looking at the (mis) fortunes of others while listening to music. Psychol. Music 42, 251-268. doi: $10.1177 / 0305735612466166$

Baumgartner, T., Lutz, K., Schmidt, C. F., and Jancke, L. (2006). The emotional power of music: how music enhances the feeling of affective pictures. Brain Res. 1075, 151-164. doi: 10.1016/j.brainres.2005.12.065

Bradley, B. P., and Lang, P. J. (2000a). Affective reactions to acoustic stimuli. Psychophysiology 37, 204-215. doi: 10.1111/1469-8986.3720204

Bradley, M. M., and Lang, P. J. (2000b). "Measuring emotion: behavior, feeling, and physiology," in Cognitive Neuroscience of Emotion, eds R. D. R. Lane, L. Nadel, G. L. Ahern, J. Allen, and A. W. Kaszniak (Oxford University Press), 25-49.

Bradley, M. M., Cuthbert, B. N., and Lang, P. J. (1990). Startle reflex modification: emotion or attention. Psychophysiology 27, 513-522. doi: 10.1111/j.14698986.1990.tb01966.x

Bradley, M. M., and Lang, P. J. (2007). The International Affective Digitized Sounds (2nd Edition; IADS-2): affective ratings of sounds and instruction manual. Gainesville, Fl.: University of Florida, NIMH Center for the Study of Emotion and Attention.

Bröckelmann, A. K., Steinberg, C., Elling, L., Zwanzger, P., Pantev, C., and Junghofer, M. (2011). Emotion-associated tones attract enhanced attention at early auditory processing: magnetoencephalographic correlates. J. Neurosci. 31, 7801-7810. doi: 10.1523/JNEUROSCI.6236-10.2011

Brosch, T., Pourtois, G., and Sander, D. (2010). The perception and categorisation of emotional stimuli: a review. Cogn. Emot. 24, 377-400. doi: 10.1080/02699930902975754

Brouwer, A.-M., van Wouwe, N., Mühl, C., van Erp, J., and Toet, A. (2013). Perceiving blocks of emotional pictures and sounds: effects on physiological variables. Front. Hum. Neurosci. 7, 1-10. doi: 10.3389/fnhum.2013.00295

Bruneau, N., Roux, S., Cléry, H., Rogier, O., Bidet-Caulet, A., and Barthélémy, C. (2013). Early neurophysiological correlates of vocal versus non-vocal sound processing in adults. Brain Res. 1528, 20-27. doi: 10.1016/j.brainres.2013.06.008 Bublatzky, F., Gerdes, A. B. M., and Alpers, G. W. (2014a). The persistence of socially instructed threat: two threat-of-shock studies. Psychophysiology 51, 1005-1014. doi: $10.1111 /$ psyp.12251

Bublatzky, F., Gerdes, A. B. M., White, A. J., Riemer, M., and Alpers, G. W. (2014b). Social and emotional relevance in face processing: happy faces of future interaction partners enhance the LPP. Front. Hum. Neurosci., 8:493. doi: 10.3389/fnhum.2014.00493

Bulkin, D. A., and Groh, J. M. (2006). Seeing sounds: visual and auditory interactions in the brain. Curr. Opin. Neurobiol. 16, 415-419. doi: 10.1016/j.conb.2006.06.008 Collignon, O., Girard, S., Gosselin, F., Roy, S., Saint-Amour, D., Lassonde, M., et al. (2008). Audio-visual integration of emotion expression. Brain Res. 1242, 126-135. doi: 10.1016/j.brainres.2008.04.023

Costafreda, S. G., Brammer, M. J., David, A. S., and Fu, C. H. (2008). Predictors of amygdala activation during the processing of emotional stimuli: a meta-analysis of 385 PET and fMRI studies. Brain Res. Rev. 58, 57-70. doi: 10.1016/j.brainresrev.2007.10.012

Cox, T. J. (2008a). The effect of visual stimuli on the horribleness of awful sounds. Appl. Acoust. 69, 691-703. doi: 10.1016/j.apacoust.2007.02.010

Cox, T. J. (2008b). Scraping sounds and disgusting noises. Appl. Acoust. 69, 11951204. doi: 10.1016/j.apacoust.2007.11.004

Craske, M. G., Hermans, D., and Vansteenwegen, D. (Eds). (2006). Fear and Learning: From Basic Processes to Clinical Implications. Washington DC: American Psychological Association.

Czigler, I., Cox, T. J., Gyimesi, K., and Horvath, J. (2007). Event-related potential study to aversive auditory stimuli. Neurosci. Lett. 420, 251-256. doi: 10.1016/j.neulet.2007.05.007

de Gelder, B., and Bertelson, P. (2003). Multisensory integration, perception and ecological validity. Trends Cogn. Sci. 7, 460-467. doi: 10.1016/j.tics.2003.08.014

de Gelder, B., and Vroomen, J. (2000). The perception of emotions by ear and eye. Cogn. Emot. 14, 289-311. doi: 10.1080/026999300378824

Dominguez-Borras, J., Garcia-Garcia, M., and Escera, C. (2008a). Emotional context enhances auditory novelty processing: behavioural and electrophysiological evidence. Eur. J. Neurosci. 28, 1199-1206. doi: 10.1111/j.1460-9568.2008.06411.x

Dominguez-Borras, J., Garcia-Garcia, M., and Escera, C. (2008b). Negative emotional context enhances auditory novelty processing. Neuroreport 19, 503-507. doi: 10.1097/WNR.0b013e3282f85bec 
Eisenbarth, H., Gerdes, A. B. M., and Alpers, G. W. (2011). Motor-Incompatibility of facial reactions. The influence of valence and stimulus content on voluntary facial reactions. J. Psychphysiol. 25, 124-130. doi: 10.1027/0269-8803/a000048

Ernst, M. O., and Bülthoff, H. H. (2004). Merging the senses into a robust percept. Trends Cogn. Sci. 8, 162-169. doi: 10.1016/j.tics.2004.02.002

Fecteau, S., Belin, P., Joanette, Y., and Armony, J. L. (2007). Amygdala responses to nonlinguistic emotional vocalizations. Neuroimage 36, 480-487. doi: 10.1016/j.neuroimage.2007.02.043

Ferrari, V., Mastria, S., and Bruno, N. (2013). Crossmodal interactions during affective picture processing. PLoS ONE 9:e89858. doi: 10.1371/journal.pone.0089858.g001

Focker, J., Gondan, M., and Roder, B. (2011). Preattentive processing of audiovisual emotional signals. Acta Psychol. 137, 36-47. doi: 10.1016/j.actpsy.2011. 02.004

Francis, S., Rolls, E. T., Bowtell, R., McGlone, F., O’Doherty, J., Browning, A., et al. (1999). The representation of pleasant touch in the brain and its relationship with taste and olfactory areas. Neuroreport 10, 453-459. doi: 10.1097/00001756199902250-00003

Gerdes, A. B. M., and Alpers, G. W. (2014). You see what you fear: spiders gain preferential access to conscious perception in spider-phobic patients. J. Exp. Psychopathol. 5, 14-28. doi: 10.5127/jep.033212

Gerdes, A. B. M., Alpers, G. W., and Pauli, P. (2008). When spiders appear suddenly: spider phobic patients are distracted by task-irrelevant spiders. Behav. Res. Ther. 46, 174-187. doi: 10.1016/j.brat.2007.10.010

Gerdes, A. B. M., Pauli, P., and Alpers, G. W. (2009). Toward and away from spiders: eye-movements in spider-fearful participants. J. Neural Transm. 116, 725-733. doi: $10.1007 /$ s00702-008-0167-8

Gerdes, A. B. M., Wieser, M. J., Alpers, G. W., Strack, F., and Pauli, P. (2012). Why do you smile at me while I'm in pain? - Pain selectively modulates voluntary facial muscle responses to happy faces. Int. J. Psychophysiol. 85, 161-167. doi: 10.1016/j.ijpsycho.2012.06.002

Gerdes, A. B. M., Wieser, M. J., Bublatzky, F., Kusay, A., Plichta, M. M., and Alpers, G. W. (2013). Emotional sounds modulate early neural processing of emotional pictures. Front. Psychol. 4:741. doi: 10.3389/fpsyg.2013.00741

Gerdes, A. B. M., Wieser, M. J., Mühlberger, A., Weyers, P., Alpers, G. W., Plichta, M. M., et al. (2010). Brain activations to emotional pictures are differentially associated with valence and arousal ratings. Front. Hum. Neurosci. 4:1. doi 10.3389/fnhum.2010.00175

Gottfried, J. A., O’Doherty, J., and Dolan, R. J. (2002). Appetitive and aversive olfactory learning in humans studied using event-related functional magnetic resonance imaging. J. Neurosci. 22, 10829-10837.

Hanser, W. E., and Mark, R. E. (2013). Music influences ratings of the affect of visual stimuli. Psychological. Topics. 22, 305-324.

Harrison, N. R., and Davies, S. J. (2013). Modulation of spatial attention to visual targets by emotional environmental sounds. Psychol. Neurosci. 6, 247-251. doi: 10.3922/j.psns.2013.3.02

Jessen, S., and Kotz, S. A. (2011). The temporal dynamics of processing emotions from vocal, facial, and bodily expressions. Neuroimage 58, 665-674. doi 10.1016/j.neuroimage.2011.06.035

Jessen, S., and Kotz, S. A. (2013). On the role of crossmodal prediction in audiovisual emotion perception. Front. Hum. Neurosci. 7, 1-7. doi: 10.3389/fnhum.2013.00369

Jessen, S., Obleser, J., and Kotz, S. A. (2012). How bodies and voices interact in early emotion perception. PLoS ONE 7:e36070. doi: 10.3389/fnhum.2013. 00369

Juslin, P. N., and Laukka, P. (2003). Communication of emotions in vocal expression and music performance: different channels, same code? Psychol. Bull. 129, 770814. doi: 10.1037/0033-2909.129.5.770

Keil, A., Bradley, M. M., Junghöfer, M., Russmann, T., Lowenthal, W., and Lang, P. J. (2007). Cross-modal attention capture by affective stimuli: evidence from event-related potentials Cogn. Affect. Behav. Neurosci. 7, 18-24. doi: 10.3758/CABN.7.1.18

Kislyuk, D. S., Möttönen, R., and Sams, M. (2008). Visual processing affects the neural basis of auditory discrimination. J. Cogn. Neurosci. 2175-2184. doi: 10.1162/jocn.2008.20152

Klasen, M., Chen, Y.-H., and Mathiak, K. (2012). Multisensory emotions: perception, combination and underlying neural processes. Rev. Neurosci. 23, 381-392. doi: 10.1515/revneuro-2012-0040
Klinge, C., Röder, B., and Büchel, C. (2010). Increased amygdala activation to emotional auditory stimuli in the blind. Brain 133, 1729-1736. doi: 10.1093/brain/awq102

Kreifelts, B., Ethofer, T., Grodd, W., Erb, M., and Wildgruber, D. (2007). Audiovisual integration of emotional signals in voice and face: an event-related fMRI study. Neuroimage 37, 1445-1456. doi: 10.1016/j.neuroimage.2007.06.020

Kumar, S., von Kriegstein, K., Friston, K., and Griffiths, T. D. (2012). Features versus feelings: dissociable representations of the acoustic features and valence of aversive sounds. J. Neurosci. 32, 14184-14192. doi: 10.1523/JNEUROSCI.175912.2012

Lang, P. J. (1995). The emotion probe: studies of motivation and attention. Am. Psychol. 50, 372-385. doi: 10.1037/0003-066X.50.5.372

Lang, P. J., and Bradley, M. M. (2010). Emotion and the motivational brain. Biol. Psychol. 84, 437-450. doi: 10.1016/j.biopsycho.2009.10.007

Lang, P. J., Bradley, M. M., and Cuthbert, B. (2008). International affective picture system (IAPS): Instruction manual and affective ratings. Technical Report A-7, The Center for Research in Psychophysiology. doi: 10.1016/j.biopsych.2010.04.020

Lang, P. J., Bradley, M. M., and Cuthbert, B. N. (1990). Emotion, attention, and the startle reflex. Psychol. Rev. 97, 377-395. doi: 10.1037/0033-295X.97.3.377

Lang, P. J., Bradley, M. M., and Cuthbert, B. N. (1998). Emotion, motivation, and anxiety: brain mechanisms and psychophysiology. Biol. Psychiatry 44, 1248-1263. doi: 10.1016/S0006-3223(98)00275-3

Lang, P. J., Bradley, M. M., and Cuthbert, M. M. (1997). "Motivated attention: affect, activation and action,". in Attention and Orienting: Sensory and Motivational Processes, eds P. J. Lang, R. F. Simons, and M. T. Balaban (Hillsdale, NJ: Lawrence Erlbaum Associates), 97-135.

LeDoux, J. E. (2000). Emotion circuits in the brain. Annu. Rev. Neurosci. 23, 155-184. doi: 10.1146/annurev.neuro.23.1.155

Logeswaran, N., and Bhattacharya, J. (2009). Crossmodal transfer of emotion by music. Neurosci. Lett. 455, 129-133. doi: 10.1016/j.neulet.2009.03.044

Marin, A., Gingras, B., and Bhattacharya, J. (2012). Crossmodal transfer of arousal, but not pleasantness, from the musical to the visual domain. Emotion 12, 618-631. doi: $10.1037 / \mathrm{a} 0025020$

Maurage, P., and Campanella, S. (2013). Experimental and clinical usefulness of crossmodal paradigms in psychiatry: an illustration from emotional processing in alcohol-dependence. Front. Hum. Neurosci. 7:394. doi: 10.3389 /fnhum.2013.00394

McGurk, H., and MacDonald, J. (1976). Hearing lips and seeing voices. Nature 264, 746-748. doi: 10.1038/264746a0

Meyer, M., Zysset, S., von Cramon, D. Y., and Alter, K. (2005). Distinct fMRI responses to laughter, speech, and sounds along the human peri-sylvian cortex. Cogn. Brain Res. 24, 291-306. doi: 10.1016/j.cogbrainres.2005.02.008

Neumann, R., Hess, M., Schulz, S. M., and Alpers, G. W. (2005). Automatic behavioural responses to valence: evidence that facial action is facilitated by evaluative processing. Cogn. Emot. 19, 499-513. doi: 10.1080/02699930512331 392562

Nishijo, H., Ono, T., and Nishino, H. (1988). Single neuron responses in amygdala of alert monkey during complex sensory stimulation with affective significance. J. Neurosci. 8, 3570-3583.

O’Doherty, J., Rolls, E. T., Francis, S., Bowtell, R., and McGlone, F. (2001). Representation of pleasant and aversive taste in the human brain. J. Neurophysiol. 85 1315-1321.

Öhman, A. (2005). The role of the amygdala in human fear: automatic detection of threat. Psychoneuroendocrinology 30, 953-958. doi: 10.1016/j.psyneuen.2005.03.019

Öhman, A., and Wiens, S. (2003). "On the automaticity of autonomic responses in emotion: an evolutionary perspective," in Handbook of Affective Sciences, eds R. J. Davidson, K. R. Scherer, and H. H. Goldsmith (New York: Oxford University Press), 256-275.

Partalaa, T., and Surakka, V. (2003). Pupil size variation as an indication of affective processing. Int. J. Hum. Comput. Stud. 59, 185-198. doi: 10.1016/S10715819(03)00017-X

Paulmann, S., and Pell, M. D. (2011). Is there an advantage for recognizing multimodal emotional stimuli? Motiv. Emot. 35, 192-201. doi: 10.1007/s11031-0119206-0

Pause, B. (2012). Processing of body odor signals by the human brain. Chemosens. Percept. 5, 55-63. doi: 10.1007/s12078-011-9108-2 
Peschard, V., Maurage, P., and Philippot, P. (2014). Towards a cross-modal perspective of emotional perception in social anxiety: review and future directions. Front. Hum. Neurosci. 8:322. doi: 10.3389/fnhum.2014.00322

Pessoa, L. (2005). To what extent are emotional visual stimuli processed without attention and awareness? Curr. Opin. Neurobiol. 15, 188-196. doi: 10.1016/j.conb.2005.03.002

Pessoa, L., McKenna, M., Gutierrez, E., and Ungerleider, L. G. (2002). Neural processing of emotional faces requires attention. Proc. Natl. Acad. Sci. U.S.A. 99, 11458-11463. doi: 10.1073/pnas.172403899

Phan, K. L., Wager, T., Taylor, S. F., and Liberzon, I. (2002). Functional neuroanatomy of emotion: a meta-analysis of emotion activation studies in PET and fMRI. Neuroimage 16, 331-348. doi: 10.1006/nimg.2002.1087

Pittig, A., Brand, M., Pawlikowski, M., and Alpers, G. W. (2014). The cost of fear: avoidant decision making in a spider gambling task. J. Anxiety Disord. 28, 326-334. doi: 10.1016/j.janxdis.2014.03.001

Plichta, M. M., Gerdes, A. B., Alpers, G. W., Harnisch, W., Brill, S., Wieser, M. J., et al. (2011). Auditory cortex activation is modulated by emotion: a functional near-infrared spectroscopy (fNIRS) study. Neuroimage 55, 1200-1207. doi: 10.1016/j.neuroimage.2011.01.011

Plichta, M. M., Schwarz, A. J., Grimm, O., Morgen, K., Mier, D., Haddad, L., et al. (2012). Test-retest reliability of evoked bold signals from a cognitive-emotive fmri test battery. Neuroimage 60, 1746-1758. doi: 10.1016/j.neuroimage.2012.01.129

Pourtois, G., Dan, E. S., Grandjean, D., Sander, D., and Vuilleumier, P. (2005). Enhanced extrastriate visual response to bandpass spatial frequency filtered fearful faces: time course and topographic evoked-potentials mapping. Hum. Brain Mapp. 26, 65-79. doi: 10.1002/hbm.20130

Pourtois, G., and Dhar, M. (2013). Integration of face and voice during emotion perception: is there anything gained for the perceptual system beyond stimulus modality redundancy," in Integrating Face and Voice in Person Perception, eds P. Belin, S. Campanella, and T. Ethofer (New York: Springer), 181-206. doi: 10.1007/978-1-4614-3585-3_10

Pourtois, G., Schettino, A., and Vuilleumier, P. (2012). Brain mechanisms for emotional influences on perception and attention: what is magic and what is not. Biol. Psychol. 92, 492-512. doi: 10.1016/j.biopsycho.2012.02.007

Rigoulot, S., and Pell, M. D. (2012). Seeing emotion with your ears: emotional prosody implicitly guides visual attention to faces. PLOS ONE 7:e30740. doi: 10.1371/journal.pone.0030740

Royet, J. P., Zald, D., Versace, R., Costes, N., Lavenne, F., Koenig, O., et al. (2000). Emotional responses to pleasant and unpleasant olfactory, visual, and auditory stimuli: a positron emission tomography study. J. Neurosci. 20, 7752-7759.

Ruys, K. I., and Stape, D. A. (2008). Emotion elicitor or emotion messenger? Subliminal priming reveals two faces of facial fxpression. Psychol. Sci. 19, 593-600. doi: 10.1111/j.1467-9280.2008.02128.x

Sabatinelli, D., Fortune, E. E., Li, Q., Siddiqui, A., Krafft, C., Oliver, W. T., et al. (2011). Emotional perception: meta-analyses of face and natural scene processing. Neuroimage 54, 2524-2533. doi: 10.1016/j.neuroimage.2010.10.011

Sander, D., Grafman, J., and Zalla, T. (2003). The human amygdala: an evolved system for relevance detection. Rev. Neurosci. 14, 303-316. doi 10.1515/REVNEURO.2003.14.4.303

Scharpf, K. R., Wendt, J., Lotze, M., and Hamm, A. O. (2010). The brain's relevance detection network operates independently of stimulus modality. Behav. Brain Res. 210, 16-23. doi: 10.1016/j.bbr.2010.01.038

Scherer, L. D., and Larsen, R. J. (2011). Cross-modal evaluative priming: emotional sounds influence the processing of emotion words. Emotion 11, 203-208. doi: $10.1037 / \mathrm{a} 0022588$

Schupp, H., Stockburger, J., Bublatzky, F., Junghöfer, M., and Weike, A. I. (2007). Explicit attention interferes with selective emotion processing in human extrastriate cortex. BMC Neurosci. 8:16. doi: 10.1186/1471-2202-8-16

Schupp, H. T., Junghoefer, M., Weike, A. I., and Hamm, A. O. (2003a). Attention and emotion. An ERP analysis of facilitated emotional stimulus processing. Neuroreport 14, 1107-1110. doi: 10.1097/00001756-200306110-00002

Schupp, H. T., Junghöfer, M., Weike, A. I., and Hamm, A. O. (2003b). Emotional facilitation of sensory processing in the visual cortex. Psychol. Sci. 14, 7-13. doi: 10.1111/1467-9280.01411

Schupp, H. T., Stockburger, J., Bublatzky, F., Junghöfer, M., Weike, A. I., and Hamm, A. O. (2008). The selective processing of emotional visual stimuli while detecting auditory targets: an ERP analysis. Brain Res. 1230, 168-176. doi: 10.1016/j.brainres.2008.07.024
Shams, L., and Kim, R. (2010). Crossmodal influences on visual perception. Phys. Life Rev, 7, 269-284. doi: 10.1016/j.plrev.2010.04.006

Shinkareva, S. V., Wang, J., Kim, J., Facciani, M. J., Baucom, L. B., and Wedell, D. H. (2014). Representations of modality-specific affective processing for visual and auditory stimuli derived from functional magnetic resonance imaging data. Hum. Brain Mapp. 35, 3558-3568. doi: 10.1002/hbm.22421

Spence, C. (2007). Audiovisual mulltisensory integration. Acoust. Sci. Technol. 28, 61-70. doi: 10.1250/ast.28.61

Stein, B. E., and Meredith, M. A. (1993). The Merging of the Senses. Cambridge, MA: The MIT Press.

Stekelenburg, J. J., and Vroomen, J. (2007). Neural correlates of multisensory integration of ecologically valid audiovisual events. J. Cogn. Neurosci. 19, 1964-1973. doi: 10.1162/jocn.2007.19.12.1964

Stienen, B. M. C., Tanaka, A., and De Gelder, B. (2011). Emotional voice and emotional body postures influence each other independently of visual awareness. PLoS ONE 6:e25517. doi: 10.1371/journal.pone.0025517

Straube, T., Mentzel, H.-J., and Miltner, W. H. R. (2006). Neural mechanisms of automatic and direct processing of phobogenic stimuli in specific phobia. Biol. Psychiatry 59, 162-170. doi: 10.1016/j.biopsych.2005.06.013

Sugimoto, S., Nittono, H., and Hori, T. (2007). Visual emotional context modulates brain potentials elicited by unattended tones. Int. J. Psychophysiol. 66, 1-9. doi: 10.1016/j.ijpsycho.2007.05.007

Surakka, V., Tenhunen-Eskelinen, M., Hietanen, J. K., and Sams, M. (1998). Modulation of human auditory information processing by emotional visual stimuli. Cogn. Brain Res. 7, 159-163. doi: 10.1016/S0926-6410(98) 00021-4

Taffou, M., Chapoulie, E., David, A., Guerchouche, R., Drettakis, G., and ViaudDelmon, I. (2012). Auditory-visual integration of emotional signals in a virtual environment for cynophobia. Ann. Rev. Cyber. Telemed. 181, 238-242.

Tartar, J. L., de Almeida, K., McIntosh, R. C., Rosselli, M., and Nash, A. J. (2012). Emotionally negative pictures increase attention to a subsequent auditory stimulus. Int. J. Psychophysiol. 83, 36-44. doi: 10.1016/j.ijpsycho.2011.09.020

Thierry, G., and Roberts, M. V. (2007). Event-related potential study of attention capture by affective sounds. Neuroreport 18, 245-248. doi: 10.1097/WNR.0b013e328011dc95

Tonoike, M., Yoshida, T., Sakuma, H., and Wang, L.-Q. (2013). fMRI measurement of the integrative effects of visual and chemical senses stimuli in humans. J. Integr. Neurosci. 12, 369-384. doi: 10.1142/S0219635213500222

Viinikainen, M., Katsyri, J., and Sams, M. (2012). Representation of perceived sound valence in the human brain. Hum. Brain Mapp. 33, 2295-2305. doi: 10.1002/hbm. 21362

Vroomen, J., Driver, J., and de Gelder, B. (2001). Is cross-modal integration of emotional expressions independent of attentional resources? Cogn. Affect. Behav. Neurosci. 1, 382-387. doi: 10.3758/CABN.1.4.382

Vuilleumier, P. (2005). How brains beware: neural mechanisms of emotional attention. Trends Cogn. Sci. 9, 585-594. doi: 10.1016/j.tics.2005.10.011

Wangelin, B. C., Bradley, M. M., Kastner, A., and Lang, P. J. (2012). Affective engagement for facial expressions and emotional scenes: the influence of social anxiety. Biol. Psychol. 91, 103-110. doi: 10.1016/j.biopsycho.2012.05.002

Watson, R., Latinus, M., Noguchi, T., Garrod, O., Crabbe, F., and Belin, P. (2013). Dissociating task difficulty from incongruence in face-voice emotion integration. Front. Hum. Neurosci. 7:744. doi: 10.3389/fnhum.2013.00744

Weymar, M., Gerdes, A. B. M., Löw, A., Alpers, G., and Hamm, A. O. (2013). Specific fear modulates attentional selectivity during visual search: electrophysiological insights from the N2pc. Psychophysiology 50, 139-148. doi: 10.1111/psyp.12008

Wickens, C. D. (2002). Multiple resources and performance prediction. Theor. Issues Ergon. Sci. 3, 159-177. doi: 10.1080/14639220210123806

Wieser, M. J., Gerdes, A. B. M., Greiner, R., Reicherts, P., and Pauli, P. (2012a). Tonic pain grabs attention, but leaves the processing of facial expressions intact evidence from event-related brain potentials. Biol. Psychol. 90, 242-248. doi: 10.1016/j.biopsycho.2012.03.019

Wieser, M. J., Gerdes, A. B. M., Reicherts, P., and Pauli, P. (2014). Mutual influences of pain and emotional face processing. Front. Psychol. 5:1160. doi: 10.3389/fpsyg.2014.01160

Wieser, M. J., McTeague, L. M., and Keil, A. (2012b). Competition effects of threatening faces in social anxiety. Emotion 12, 1050-1060. doi: 10.1037/a0027069 Yamashita, H., Okamoto, Y., Morinobu, S., Yamawaki, S., and Kahkonen, S. (2005). Visual emotional stimuli modulation of auditory sensory gating studied 
by magnetic P50 suppression. Eur. Arch. Psychiatry Clin. Neurosci. 255, 99-103. doi: 10.1007/s00406-004-0538-6

Yokosawa, K., Pamilo, S., Hirvenkari, L., Hari, R., and Pihko, E. (2013). Activation of auditory cortex by anticipating and hearing emotional sounds: an MEG study PLoS ONE 8:e80284. doi: 10.1371/journal.pone.0080284

Zald, D. H. (2003). The human amygdala and the emotional evaluation of sensory stimuli. Brain Res. Brain Res. Rev. 41, 88-123. doi: 10.1016/S0165-0173(02) 00248-5

Zeelenberg, R., and Bocanegra, B. R. (2010). Auditory emotional cues enhance visual perception. Cognition 115, 202-206. doi: 10.1016/j.cognition.2009.12.004

Conflict of Interest Statement: The authors declare that the research was conducted in the absence of any commercial or financial relationships that could be construed as a potential conflict of interest.
Received: 14 August 2014; accepted: 06 November 2014; published online: 01 December 2014.

Citation: Gerdes ABM, Wieser MJ and Alpers GW (2014) Emotional pictures and sounds: a review of multimodal interactions of emotion cues in multiple domains. Front. Psychol. 5:1351. doi: 10.3389/fpsyg.2014.01351

This article was submitted to Emotion Science, a section of the journal Frontiers in Psychology.

Copyright (C) 2014 Gerdes, Wieser and Alpers. This is an open-access article distributed under the terms of the Creative Commons Attribution License (CC BY).

The use, distribution or reproduction in other forums is permitted, provided the original author(s) or licensor are credited and that the original publication in this journal is cited, in accordance with accepted academic practice. No use, distribution or reproduction is permitted which does not comply with these terms. 